\title{
C. elegans models of Marfan and Marfan-like Syndromes reveal trafficking defects of the type II TGF $\beta$ receptor as a potential novel disease mechanism
}

\author{
Jing Lin, Mehul Vora, Nanci S Kane, Ryan J Gleason, and Richard W Padgett
}

The article has been withdrawn by the authors due to concerns from one of the authors regarding information being available prior to publication. Therefore, the authors do not wish this work to be cited as reference for the project. 